\title{
Outcome of Gastric Cancer Surgery in Elderly Patients
}

\author{
Min Sung Kim and Sungsoo Kim \\ Department of Surgery, School of Medicine, Chosun University, Gwangju, Korea
}

Purpose: Owing to increased life expectancy, the number of elderly patients with gastric cancer has increased. This study aimed to identify the outcomes of gastric cancer patients aged 80 years or older through comparison of their clinicopathological characteristics, surgical outcomes, and oncologic outcomes.

Materials and Methods: Between January 2006 and December 2013, the records of 478 patients who underwent surgery for gastric cancer were retrospectively evaluated. Patients were divided into two groups: patients $<80$ years old $(n=446)$ and patients $\geq 80$ years old $(n=32)$.

Results: There were no significant differences in sex, body mass index, length of hospital stay, duration of surgery, depth of invasion, nodal metastasis, histologic type, or tumor size between the two groups. However, significant differences were found for the American Society of Anesthesiologist score and the serum albumin level between the two groups. Postoperative morbidity, mortality, disease-free survival, and recurrence rate did not differ between curatively resected patients in the two groups.

Conclusions: In elderly patients with gastric cancer, active treatment including radical gastrectomy is necessary.

Key Words: Elderly; Treatment outcome; Stomach neoplasms; Complications; Gastrectomy

\section{Introduction}

With increased life expectancy and an increasing elderly population, the prevalence of various types of cancer is increasing. ${ }^{1}$ The older population ( $>65$ years old) in South Korea steadily increased from $12.2 \%$ in 2013 to $13.2 \%$ in 2015, and, by 2020, the average life expectancy is expected to be 79.5 years and 85.7 years for men and women, respectively. ${ }^{2}$ Gastric cancer is caused by environmental factors and the accumulation of specific genetic alterations. The increase in average age has resulted in an increased incidence of gastric cancer in the older population.

Although the incidence of gastric cancer has recently de-

\section{Correspondence to: Sungsoo Kim}

Department of Surgery, School of Medicine, Chosun University, 309

Pilmun-daero, Dong-gu, Gwangju 61452, Korea

Tel: +82-62-220-3010, Fax: +82-62-228-3441

E-mail: sungsoo73@chosun.ac.kr

Received September 8, 2016

Revised October 5, 2016

Accepted October 8, 2016 creased, as of 2013, it was the most frequently occurring cancer in men and the 4th most frequent in women in South Korea after thyroid, breast, and colorectal cancer. ${ }^{4}$ Moreover, after lung and liver cancer, the rate of mortality due to gastric cancer is the 3rd highest in South Korea. ${ }^{5}$ However, with the increased chance of early diagnosis through routine screening, the prognosis of gastric cancer is thought to be much more favorable. ${ }^{6}$

According to a Japanese study, surgical treatment was associated with a better prognosis than supportive care in gastric cancer patients $\geq 80$ years old. $^{7}$ Nevertheless, the elderly population often has underlying conditions such as cardiovascular, cerebrovascular, kidney, and pulmonary diseases, or organs with reduced functionality. Therefore, the rate of mortality or complications is higher in older patients, ${ }^{8,9}$ and surgeons tend to avoid performing surgeries on older patients.

This study aims to assess surgical outcomes in gastric cancer patients $\geq 80$ years old by comparing clinicopathological characteristics and oncologic/surgical outcomes between patients $<80$ years old and $\geq 80$ years old. 


\section{Materials and Methods}

This was a retrospective study of 478 patients with gastric cancer who underwent surgery at Chosun University Hospital between January 2006 and December 2013. Patients were divided into two groups according to age: $<80$ years (young group, $n=446$ ) and $\geq 80$ years (elderly group, $n=32$ ). Forty-three patients underwent non-curative surgery (young group, $n=42$; elderly group, $\mathrm{n}=1$ ).

D1+ or D2 lymph node dissection was performed according to the standards of the Japanese Gastric Cancer Association. ${ }^{10}$ The surgical method was selected based on the location of the lesion and the preference of the surgeon. The resection margin was checked on frozen biopsy. Curative resection (R0) meant

Table 1. Demographic characteristics of the patients

\begin{tabular}{|c|c|c|c|}
\hline Variable & Young group $(\mathrm{n}=446)$ & Elderly group (n=32) & P-value \\
\hline Gender & & & 0.183 \\
\hline Male & $291(65.2)$ & $17(53.1)$ & \\
\hline Female & $155(34.8)$ & $15(46.9)$ & \\
\hline Age (yr) & $60.4 \pm 11.5$ & $82.3 \pm 2.1$ & $<0.001$ \\
\hline Body mass index $\left(\mathrm{kg} / \mathrm{m}^{2}\right)$ & $23.1 \pm 3.1$ & $22.2 \pm 2.7$ & 0.102 \\
\hline Preoperative serum albumin level (g/dl) & $4.5 \pm 0.5$ & $4.1 \pm 0.5$ & $<0.001$ \\
\hline ASA score & & & $<0.001$ \\
\hline 1 & $135 / 404(33.4)$ & $1 / 31(3.2)$ & \\
\hline 2 & 236/404 (58.4) & $22 / 31(71.0)$ & \\
\hline 3 & $33 / 404(8.2)$ & $8 / 31(25.8)$ & \\
\hline Histology type & & & 0.506 \\
\hline Differentiated & $314 / 404(77.7)$ & $26 / 31(83.9)$ & \\
\hline Undifferentiated & $90 / 404(22.3)$ & $5 / 31(16.1)$ & \\
\hline T stage & & & 0.173 \\
\hline $\mathrm{T} 1$ & $263 / 404(65.1)$ & $16 / 31(51.6)$ & \\
\hline $\mathrm{T} 2, \mathrm{~T} 3, \mathrm{~T} 4$ & $141 / 404(34.9)$ & $15 / 31(48.4)$ & \\
\hline $\mathrm{N}$ stage & & & 1.000 \\
\hline No & $303 / 404(75.0)$ & 24/31 (77.4) & \\
\hline $\mathrm{N} 1, \mathrm{~N} 2, \mathrm{~N} 3$ & $101 / 404(25.0)$ & $7 / 31(22.6)$ & \\
\hline TNM stage* & & & 0.412 \\
\hline IA & $246(55.2)$ & $16(50.0)$ & \\
\hline IB & $52(11.7)$ & $4(12.5)$ & \\
\hline IIA & $35(7.8)$ & $6(18.8)$ & \\
\hline IIB & $22(4.9)$ & $2(6.3)$ & \\
\hline IIIA & $26(5.8)$ & $1(3.1)$ & \\
\hline IIIB & $16(3.6)$ & $2(6.3)$ & \\
\hline IIIC & $7(1.6)$ & 0 & \\
\hline IV & $42(9.4)$ & $1(3.1)$ & \\
\hline Tumor size (cm) & $2.3 \pm 2.2$ & $3.1 \pm 3.6$ & 0.229 \\
\hline No. of harvested lymph node & $30.1 \pm 15.1$ & $28.7 \pm 14.5$ & 0.632 \\
\hline Curability & $404(90.6)$ & $31(96.9)$ & 0.343 \\
\hline Non-curative surgery ${ }^{\dagger}$ & $42(9.4)$ & $1(3.1)$ & 0.343 \\
\hline
\end{tabular}

Values are presented as number (\%) or mean \pm standard deviation. The sum of the percentages does not equal $100 \%$ because of rounding. ASA $=$ American Society of Anesthesiologist; TNM = tumor node metastasis. ${ }^{\star}$ Classification according to the American Joint Committee on Cancer 7th edition. ${ }^{\dagger}$ Open \& close, bypass surgery, palliative total gastrectomy. 
that cancer cells were not observable under the microscope in the proximal and distal margins, including in the metastatic lymph nodes, while non-curative surgery (R1 or R2) indicated that cancer cells were visible either under the microscope or by the naked eye.

Postoperative complications were categorized based on the Clavien-Dindo classification, ${ }^{11-13}$ and complications higher than grade 3 were categorized as severe complications. Patients were followed-up every 3 months for the first 2 years, and every 6 months thereafter. Endoscopic gastroduodenoscopy and computed tomography were performed annually, and the average follow-up period was 39.6 \pm 26.3 months (range, 0 103 months).

Statistical analysis was performed using IBM SPSS Statistics ver. 21.0 (IBM Co., Armonk, NY, USA). Patient age, gender, curability, body mass index (BMI), American Society of Anesthesiologist (ASA) score, preoperative serum albumin level, type of surgery, length of stay (LOS), duration of surgery, presence of nodal metastasis, pathologic stage, tumor size, histological type, postoperative complications, and disease-free survival (DFS) were included in the analysis. The statistical tests used were Fisher's exact test, the chi-square test, and the Mann-Whitney U-test. Multivariate analysis was performed to identify risk factors of postoperative complications. DFS was calculated using the Kaplan-Meier method and compared using log rank tests. A $\mathrm{P}$-value of less than 0.05 was considered as statistically significant.

This study was approved by the Institutional Review Board of Chosun University Hospital (approval number: CHOSUN 2014-08-006).

\section{Results}

Table 1 summarizes the demographic findings of 478 patients. There were 446 patients and 32 patients in the young and elderly groups, respectively. The average age in the young group was $60.4 \pm 11.5$ years (range, 24 79 years; $\mathrm{P}<0.001$ ), while the average age in the elderly group was 82.3 \pm 2.1 years (range, 80 87 years; $\mathrm{P}<0.001$ ). The ASA score and preoperative serum albumin levels were significantly different between the groups $(\mathrm{P}<0.05)$. The preoperative serum albumin level was higher in the young group than in the elderly group $(4.5 \pm 0.5 \mathrm{~g} / \mathrm{dl}$ vs. $4.1 \pm 0.5 \mathrm{~g} / \mathrm{dl}$; $\mathrm{P}<0.001)$. Other measurements such as gender, BMI, T stage, $\mathrm{N}$ stage, pathologic stage, and tumor size were not statistically different ( $\mathrm{P}>0.05$ for each). There were 43 patients who had noncurative surgery. In the young group, there were 11 patients who underwent open and close surgery, 18 patients who underwent bypass surgery, and 13 patients who underwent palliative total gastrectomy. One patient in the elderly group underwent bypass surgery. Excluding the above 43 patients, Table 2 summarizes the surgical outcomes of the 435 patients who underwent curative resection.

There was no statistical difference between the two groups for LOS, duration of surgery, type of surgery, mortality, or type of complications ( $\mathrm{P}>0.05$ for each). Postoperative complications occurred in $27.1 \%$ (118/435) of patients who underwent curative resection. The most frequent complications were wound healing complications (15.9\%, 69/435; young group 65/404; elderly group, 4/31), and leakage complications (4.8\%, 21/435; young group 20/404; elderly group, 1/31) (Table 3).

Multivariate analysis was performed on risk factors that af-

Table 2. Surgical outcomes in patients who underwent curative resection

\begin{tabular}{lccc}
\hline \multicolumn{1}{c}{ Variable } & Young group $(\mathrm{n}=404)$ & Elderly group $(\mathrm{n}=31)$ & P-value \\
\hline Length of stay $(\mathrm{d})$ & $14.5 \pm 11.0$ & $13.7 \pm 7.3$ & 0.675 \\
Duration of surgery $(\mathrm{min})$ & $212.0 \pm 61.0$ & $200.0 \pm 52.0$ & 0.283 \\
Type of surgery & & & 1.000 \\
Subtotal gastrectomy & $328(81.2)$ & $25(80.6)$ & $6(19.4)$ \\
Total gastrectomy & $76(18.8)$ & $2(6.5)$ & 0.129 \\
Mortality rate & $7(1.7)$ & & 0.905 \\
Complications & & $23(74.2)$ & $7(22.6)$ \\
None & $294(72.8)$ & $1(3.2)$ \\
Local & $101(25.0)$ & $9(2.2)$ & \\
Systemic & &
\end{tabular}

Values are presented as mean \pm standard deviation or number (\%). 
Outcome of Gastric Cancer Surgery in Elderly

Table 3. Postoperative complications in patients who underwent curative resection

\begin{tabular}{lcc}
\hline \multicolumn{1}{c}{ Variable } & Young group $(\mathrm{n}=404)$ & Elderly group $(\mathrm{n}=31)$ \\
\hline Total complication & $110(27.2)$ & $8(25.8)$ \\
Wound problems & $65(16.1)$ & $4(12.9)$ \\
Leakage & $20(5.0)$ & $1(3.2)$ \\
Respiratory problems & $4(1.0)$ & $1(3.2)$ \\
PRC transfusion & $11(2.7)$ & $1(3.2)$ \\
Ileus & $4(1.0)$ & $1(3.2)$ \\
Intra-abdominal fluid collection & $3(0.7)$ & 0 \\
Cardiovascular problems & $3(0.7)$ & 0 \\
Severe complications (grade 3/4/5)* & $48(11.9)$ & $5(16.1)$ \\
Wound problems & $19 / 0 / 0$ & $2 / 1 / 0$ \\
Leakage & $15 / 0 / 4$ & $0 / 0 / 1$ \\
Respiratory problems & $3 / 1 / 0$ & $1 / 0 / 0$ \\
Intra-abdominal fluid collection & $2 / 0 / 1$ & $0 / 0 / 0$ \\
Cardiovascular problems & $0 / 0 / 3$ & $0 / 0 / 0$ \\
\hline
\end{tabular}

Values are presented as number (\%) or number only. $\mathrm{PRC}=$ packed red blood cells. ${ }^{\star}$ Clavien-Dindo classification grade 3 or above.

Table 4. Multivariate logistic regression results for complications

\begin{tabular}{|c|c|c|c|c|}
\hline \multirow{2}{*}{ Variable } & \multicolumn{2}{|c|}{ Complications } & \multicolumn{2}{|c|}{ Severe complications } \\
\hline & OR $(95 \% \mathrm{CI})$ & P-value & OR $(95 \% \mathrm{CI})$ & P-value \\
\hline Sex (male) & $2.0(1.2 \sim 3.3)$ & 0.005 & $2.7(1.3 \sim 5.5)$ & 0.009 \\
\hline Age $(\geq 80 \mathrm{yr})$ & & 0.454 & & 0.623 \\
\hline ASA score & & 0.034 & & 0.097 \\
\hline 1 & 1 & & 1 & \\
\hline 2 & $1.4(0.8 \sim 2.3)$ & 0.240 & & \\
\hline 3 & $2.9(1.3 \sim 6.4)$ & 0.009 & & \\
\hline STG/TG & & 0.128 & & 0.118 \\
\hline Preoperative serum albumin level & & 0.350 & & 0.193 \\
\hline
\end{tabular}

$\mathrm{OR}=$ odds ratio $\mathrm{CI}=$ confidence interval; $\mathrm{ASA}=$ American Society of Anesthesiologist $\mathrm{STG}$ = subtotal gastrectomy; $\mathrm{TG}=$ total gastrectomy.

fected postoperative complications (Table 4). Type of surgery, preoperative serum albumin level, pathologic stage, and patient age did not affect postoperative complications. However, gender and ASA score were identified as risk factors for complications. Severe complications occurred in 12.2\% (53/435) of patients (Table 4). Gender was the only risk factor for severe complications.

Fig. 1 shows the DFS of patients who underwent curative resection for gastric cancer. The mean DFS was 93.6 \pm 1.5 months (95\% confidence interval [CI], 90.6 96.9 months): patients in the young group had a mean DFS of $93.5 \pm 1.6$ months $(95 \%$ CI, 90.4 96.9 months), while patients in the elderly group had a mean DFS of 61.6 \pm 3.3 months (95\% CI, 55.2 68.0 months). There was no significant difference in the 5-year DFS rates between the two groups (young group, $88.7 \%$ vs. elderly group, 92.9\%; $\mathrm{P}=0.680)$. The recurrence rates were $8.2 \%$ (33/404) and $3.2 \%(1 / 31)$ in the young and elderly groups, respectively, with no statistical difference $(\mathrm{P}=0.496)$.

\section{Discussion}

Life expectancy and average age are steadily increasing in South Korea due to the combination of economic development, 


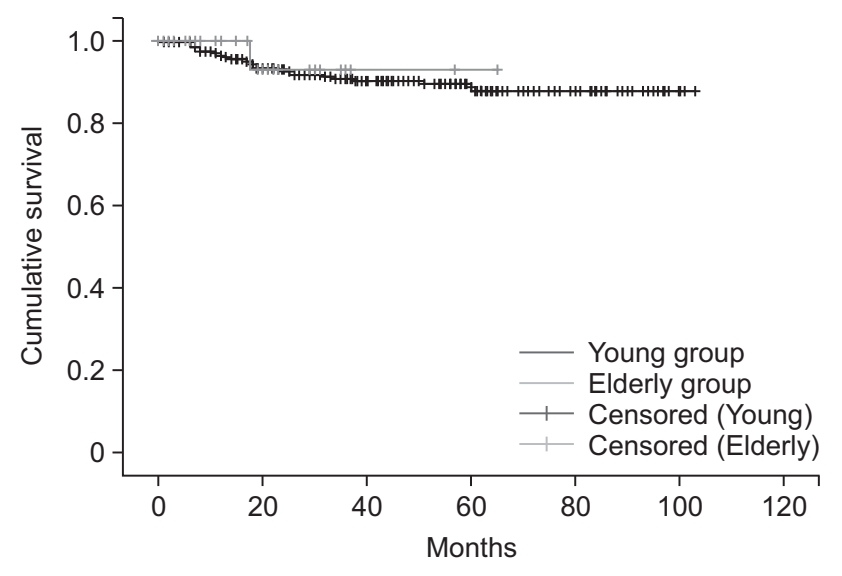

Fig. 1. Disease free survival of curative resected patients. Young group, $<80$ years old; Elderly group, $\geq 80$ years old.

balanced diet, increased quality of life, and increased interest in a healthy life. However, this increase in the average age is accompanied by a higher prevalence of various types of cancers; included among those that affect the older population is gastric cancer. Furthermore, the increased age means that there are more age-related complications. It is well known that older patients are at higher risk during surgery than younger patients. ${ }^{14}$ In gastric cancer, curative resection is associated with a survival benefit; therefore, deciding upon the appropriate treatment method for elderly gastric cancer patients is important.

The average age of the patients in this study was $62.0 \pm 12.3$ years (range, 24 87 years). These patients were divided into two groups: a young group ( $<80$ years old) and an elderly group ( $\geq 80$ years). Only $7.0 \%$ (31/445) of the entire patient cohort belonged to the elderly group. Previous studies that compared surgical outcomes between younger and older gastric cancer patients had lower age cut-offs for the older patient group than our study, and they reported no differences in surgical outcomes. ${ }^{15,16}$ One study had the same age cut-off as our study; in that study $8.7 \%(104 / 1,193)$ of the patient cohort belonged to the elderly group, similar to the current study. It reported that there were no differences in surgical outcomes between patients $<80$ years old and patients $\geq 80$ years old. ${ }^{17}$

Gastric cancer is thought to occur more frequently in men. In line with this, the current study had more male patients; however, the elderly group had a male to female ratio of $1: 1$. The increased number of women in the elderly group is probably due to the lower life expectancy of men, which is below 80 years of age, according to previous statistics. This means that they would have died from natural causes or other diseases before they reached the age cut-off in this study.

Patients with high surgical risks have an increased rate of complications, mortality, and LOS. ${ }^{18}$ Results from this study showed a lower level of preoperative serum albumin level and a higher ASA score in the elderly group, compared with the young group, and suggesting a higher surgical risk in the elderly group. However, there were no statistical differences in postoperative complications, mortality rate, or LOS between the two groups. The difference in ASA may be due to more careful preoperative assessments concerning organ function and performance status. Another possibility is that the elderly patients received more postoperative care than the younger patients.

In terms of surgical outcomes between the two groups, there were no statistical differences in $\mathrm{T}$ stage, $\mathrm{N}$ stage, pathologic stage, or the number of retrieved lymph nodes. Similarly, when curative resection was performed in gastric cancer patients aged 80 years, DFS and recurrence rates were not significantly different from those of the young group. This finding is consistent with a previous study by Takeshita et al. ${ }^{17}$

Patients who undergo gastrectomy have trouble with the consumption and digestion of food, and are often nutritionally imbalanced. This may lead to various health conditions in elderly patients who undergo gastrectomy. ${ }^{19}$ The average remaining life expectancy in the general population aged 80 years or older is 9.5 years: 8.0 years and 10.3 years in men and women, respectively. ${ }^{20}$ The mean DFS for the elderly group in this study was 61.6土 3.3 months (95\% CI, 55.2 68.0 months), which is comparable to the remaining life expectancy of the general population aged 80 years or older.

In South Korea, screening for gastric cancer is most frequently performed in people aged between 50 years and 60 years of age, and the frequency of screening decreases in older individuals. ${ }^{21}$ However, the results from our study indicate good surgical outcomes in elderly gastric cancer patients ( $\geq 80$ years); therefore, regular screening in the elderly population may be required.

This study has several limitations. First, the inherent features of a nonrandomized retrospective cohort study were inevitable. Secondly, elderly patients in this study tolerated total or subtotal gastrectomy relatively well, and demonstrated good functional status. Thirdly, this sample size was small. Therefore, it is hard to draw a definitive conclusion. Aggressive treatment may be considered even in elderly patients with gastric cancer if surgical intervention is still possible.

In conclusion, surgical outcomes between younger patients ( $<80$ years) and elderly patients ( $\geq 80$ years) were not signifi- 
cantly different. Therefore, even in elderly patients, surgical intervention may be the more promising treatment, if curative resection is possible.

\section{Acknowledgments}

This study was supported by a research grant from Chosun University, 2015.

\section{Conflicts of Interest}

No potential conflict of interest relevant to this article was reported.

\section{References}

1. Bray F, Jemal A, Grey N, Ferlay J, Forman D. Global cancer transitions according to the Human Development Index (2008-2030): a population-based study. Lancet Oncol 2012;13:790-801.

2. Korean Statistical Information Service [Internet]. Daejeon: Korean Statistical Information Service; 2016 [cited 2016 Aug 29]. Available from: http://kosis.kr/statHtml/statHtml. do?orgId=101\&tblId=DT_1B42\&conn_path=I2.

3. Tavares A, Gandra A, Viveiros F, Cidade C, Maciel J. Analysis of clinicopathologic characteristics and prognosis of gastric cancer in young and older patients. Pathol Oncol Res 2013;19: 111-117.

4. Siegel R, Naishadham D, Jemal A. Cancer statistics, 2013. CA Cancer J Clin 2013;63:11-30.

5. National Cancer Information Center [Internet]. Goyang: National Cancer Information Center; 2013 [cited 2016 Aug 22]. Available from: http://www.cancer.go.kr/mbs/cancer/subview. jsp?id=cancer_040202000000.

6. Jeong O, Park YK. Clinicopathological features and surgical treatment of gastric cancer in South Korea: the results of 2009 nationwide survey on surgically treated gastric cancer patients. J Gastric Cancer 2011;11:69-77.

7. Matsushita I, Hanai H, Kajimura M, Tamakoshi K, Nakajima T, Matsubayashi Y, et al. Should gastric cancer patients more than 80 years of age undergo surgery? Comparison with patients not treated surgically concerning prognosis and quality of life. J Clin Gastroenterol 2002;35:29-34.

8. Bittner R, Butters M, Ulrich M, Uppenbrink S, Beger HG. Total gastrectomy. Updated operative mortality and long-term survival with particular reference to patients older than 70 years of age. Ann Surg 1996;224:37-42.

9. Eguchi T, Takahashi Y, Ikarashi M, Kasahara M, Fujii M. Is extended lymph node dissection necessary for gastric cancer in elderly patients? Eur J Surg 2000;166:949-953.

10. Japanese Gastric Cancer Association. Japanese classification of gastric carcinoma: 3rd English edition. Gastric Cancer 2011;14:101-112.

11. Dindo D, Demartines N, Clavien PA. Classification of surgical complications: a new proposal with evaluation in a cohort of 6336 patients and results of a survey. Ann Surg 2004;240:205213.

12. Clavien PA, Strasberg SM. Severity grading of surgical complications. Ann Surg 2009;250:197-198.

13. Clavien PA, Barkun J, de Oliveira ML, Vauthey JN, Dindo D, Schulick RD, et al. The Clavien-Dindo classification of surgical complications: five-year experience. Ann Surg 2009;250:187-196.

14. Wu CW, Lo SS, Shen KH, Hsieh MC, Lui WY, P'eng FK. Surgical mortality, survival, and quality of life after resection for gastric cancer in the elderly. World J Surg 2000;24:465-472.

15. Pisanu A, Montisci A, Piu S, Uccheddu A. Curative surgery for gastric cancer in the elderly: treatment decisions, surgical morbidity, mortality, prognosis and quality of life. Tumori 2007;93:478-484.

16. Saidi RF, Bell JL, Dudrick PS. Surgical resection for gastric cancer in elderly patients: is there a difference in outcome? J Surg Res 2004;118:15-20.

17. Takeshita H, Ichikawa D, Komatsu S, Kubota T, Okamoto K, Shiozaki A, et al. Surgical outcomes of gastrectomy for elderly patients with gastric cancer. World J Surg 2013;37:2891-2898.

18. Sun Z, Kong XJ, Jing X, Deng RJ, Tian ZB. Nutritional risk screening 2002 as a predictor of postoperative outcomes in patients undergoing abdominal surgery: a systematic review and meta-analysis of prospective cohort studies. PLoS One 2015;10:e0132857.

19. Watanabe $M$, Iwatsuki $M$, Iwagami S, Ishimoto T, Baba Y, Baba H. Prognostic nutritional index predicts outcomes of gastrectomy in the elderly. World J Surg 2012;36:1632-1639.

20. Korean Statistical Inforamtion Service [Internet]. Daejeon: Korean Statistical Inforamtion Service; 2016 [cited 2016 Aug 22]. Available from: http://kosis.kr/statHtml/statHtml.do?orgId=10 1\&tblId=DT_1YL0201\&conn_path=I2.

21. Suh M, Choi KS, Lee YY, Park B, Jun JK. Cancer screening in Korea, 2012: results from the Korean National Cancer Screening Survey. Asian Pac J Cancer Prev 2013;14:6459-6463. 